\title{
3D CFD Simulation of the Natural Gas Emission from Damaged Pipelines
}

\author{
B. Mohseni-Gharyehsafa, V. Okati, A. Ebrahimi-Moghadam and Y. Bijarzehi*
}

\begin{abstract}
Gas pipelines are often subjected to hazardous accidents which cause gas released to the atmosphere. At the first step after the accident happening, it is very necessary to perform hazard management, for decision to take the necessary steps of loss prevention. The aim of the present study is to simulate natural gas (NG) leakage scenario form low \& medium pressure NG distribution pipelines. The natural gas is considered as pure methane with real gas behavior. The fluid properties is calculated by using RK EOS. The results analysis shows that the amount of leaked gas has a linear relation, second order relation with pressure of initial point, diameter of hole, respectively.
\end{abstract}

Index Terms-Numerical simulation, Natural gas, Real gas EOS.

\section{INTRODUCTION}

The most common and comfortable method of transporting and distributing NG are pipelines. NG pipelines are usually subjected to various damages such as mechanical or material failure, corrosion, third party activities and etc. [2] and these causes to lost and unaccounted-for (LAUF) gas [4], [5]. Several factors are involved in existence of the LAUF and among them, gas leakage is the most important factor [6]. Gas leakage causes major incidents resulting in both environmental and financial losses and consequently there needs to know about the NG leakage scenario.

The objective of this research is calculation the amount of the NG leakage. To analyze one-dimensional compressible gas flow in high pressure buried pipelines, a research was done by Nouri-Borujerdi and Ziaei-Rad [7]. The pressure perturbations generation was verified with a CFD simulation by Liu et al. [8] to study the fundamental of acoustic leak detection. A new approach to evaluate the amount of gas release was introduced by Moloudi and Abolfazli Esfahani [9]. Lu et al. [10] analyzed the theory of the one-dimensional natural gas leakage in urban medium pressure pipelines, and examined their proposed model for both steady and transient (unstable) states. Numerical simulation of isothermal and adiabatic flows as a one dimensional model was done by Kostowski and Skorek [11]. A detailed review of sonic gas flow in pipelines was presented by Keith and Crowl [12]. Monitel et al. [13] developed a mathematical model of accidental gas release. The formulation of the Montiel et al. [13] was based on isentropic Fanno theory. Cavazzuti and Corticelli [14] solved non-isentropic compressible Fanno flow in micro channel and validate it by a CFD simulation. A numerical simulation of high-pressure pipeline puncture was introduced by Oke et al. [15]. Jo and Ahn [16] developed a simple and definite model based on Fanning equation to calculate the amount of leaked gas from a hole in high-pressure gas pipelines. Luo et al. [17] proposed a mathematical model for estimating flow-rate of gas through a high-pressure damaged pipe. Liu et al. [18] verified the pressure perturbations generation by using a numerical model.

In all of previous works, no research has been conducted on NG leakage from pipelines as 3D model. In the other word, almost all of the recent studies were paid to simulate NG leakage from a close reservoir as one or two dimensional flows. Also most previous studies were satisfied only to investigate some of the effective parameters on leakage. In current work, for making the results closer to the actual scenario, is paid to simulate NG leakage process as a three-dimensional model with real gas assumption and analyze the impact of all effective parameters (pressure, hole diameter and pipe diameter) on the amount of the NG leakage.

\section{Problem Statement}

For modeling leakage, there are two situations: hole model and pipe model. In the hole model there is a hole on the lateral surface of the pipe, hole diameter is smaller than pipe diameter $(\mathrm{d} \cdot \mathrm{D})$ and gas discharges from the hole. But pipe model is a case that the diameter of the hole is equal or larger than pipe (d - D). Fig. 1 shows the model which was used in previous studies and according to this figure, pipe was considered like a tank (closed reservoir). According to Fig. 1, a pipeline is connected to a reservoir by converging nozzle. The lengths of the nozzle and the hole $(\cdot 1$ and $\cdot 2)$ are relatively small in comparison to the pipe length.
B. Mohseni-Gharyehsafa and V. Okati are with the Faculty of Mechanical Engineering, Shahrood University of technology, Shahrood, Iran

A. Ebrahimi-Moghadam is with the Young Researchers and Elite Club, Mashhad Branch, Islamic Azad University, Mashhad, Iran

V. Okati is also with Faculty of Marine Engineering, Chabahar Maritime University, Chabahar, Iran

${ }^{*}$ Y. Bijarzehi is with Saba Operation and Maintenance Company, Tehran, Iran. 


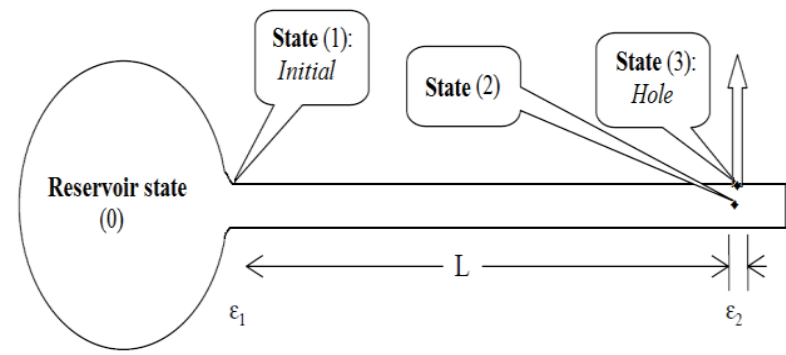

Fig. 1: A schematic of the one-dimensional model (as reservoir)

\section{GOVERNING EQUATIONS}

In this work, a steady, compressible and turbulent flow has been built to calculate the amount of NG leakage from urban distribution pipelines. The main ingredient of NG is methane (more than 90\%) and therefore equalization of methane as NG is not far from reality. The governing equations of the problem involve continuity, momentum, energy and RK equation of state (EOS). These equations are respectively expressed as follows [19]-[21]:

$$
\begin{aligned}
& \frac{\partial}{\partial x_{i}}\left(\rho u_{i}\right)=0 \\
& \frac{\partial}{\partial x_{j}}\left(\rho u_{i} u_{j}\right)=-\frac{\partial p}{\partial x_{i}}+\frac{\partial \overline{\bar{\tau}}_{i j}}{\partial x_{j}}+\frac{\partial}{\partial x_{j}}\left(-\rho \overline{u_{\imath} \dot{u}_{j}}\right) \\
& \frac{\partial}{\partial x_{i}}\left[u_{i}(\rho E+p)\right]=\frac{\partial}{\partial x_{j}}\left[k_{e f f} \frac{\partial T}{\partial x_{j}}+u_{i}\left(\overline{\bar{\tau}}_{i j}\right)_{e f f}\right] \\
& p=\frac{R T}{v-b}-\frac{a}{\sqrt{T} \cdot v(v+b)}
\end{aligned}
$$

In which, $\rho, u, p, x, \tau, E, k, T, R$ and $v$ are density, velocity, pressure, displacement, stress, energy, thermal conductivity, temperature, gas constant and specific volume, respectively. Also, $a$ and $b$ are the coefficients of the RK EOS.

\section{GeOMETRY AND GRID GENERATION}

The geometry is a pipe which a hole has been created on the lateral surface of it and NG flow is released from it. The pipe is considered as a three-dimensional model. Fig. 2 is schematic of the model considered in this work. The model of this investigation is a polyethylene pipe [22] located in the urban gas distribution system with low and medium pressure level.

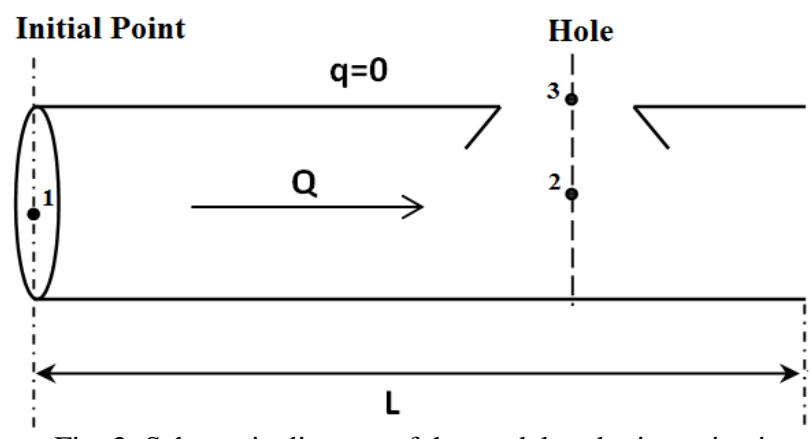

Fig. 2: Schematic diagram of the model under investigation

The grid structure has been employed for computation domain shown in Fig. 3. The unstructured mesh is used and the fine grid has been utilized near the walls as well as hole, where the highest change is expected.

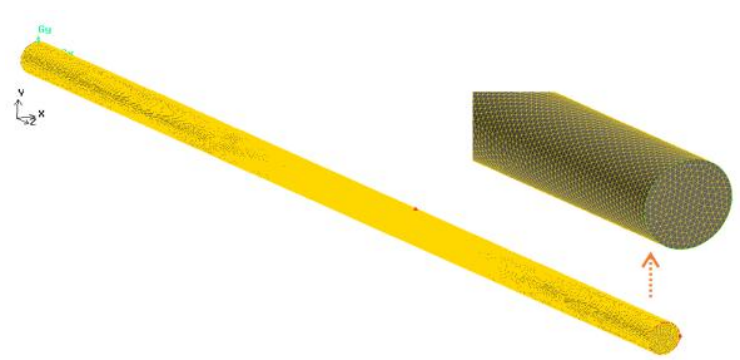

Fig. 3: The computational grid used in this study

\section{BOUNDARY CONDITIONS}

The boundary conditions which are used in the computational domain have been shown in Fig. 4. The pipe under investigation has been located in the urban distribution gas pipelines with low pressure level and relatively small pipe diameter. The inlet temperature is $288 \mathrm{~K}$ and the problem is investigated with the different amounts of beginning point pressure in the range of 3 bar to 5 bar absolute. Hole diameter is investigated in the range of $5 \mathrm{~mm}$ to $80 \mathrm{~mm}$ and pipe diameter investigated for three most common pipe sizes (with nominal diameters: $114.6 \mathrm{~mm}$, $163.6 \mathrm{~mm}$ and $204.6 \mathrm{~mm}$ ) which are used in urban gas distribution systems.

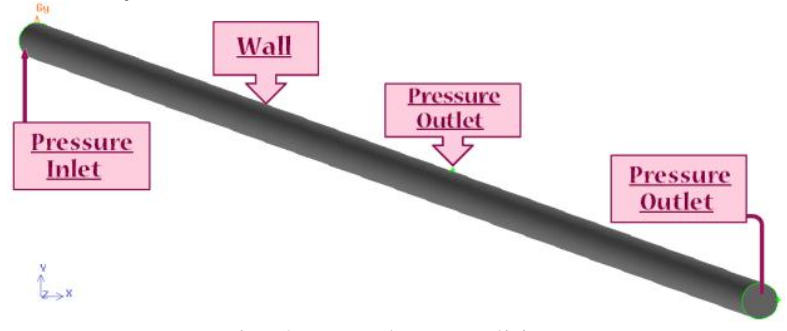

Fig. 4: Boundary conditions

\section{RESULTS AND DISCUSSION}

\section{A. Model validation}

The results of this simulation are validated by comparing with Montiel's model [13]. The model of Montiel et al. [13] was a pipe with an inner diameter of $163.6 \mathrm{~mm}$ in distribution pipelines with 5 bar initial pressure which had a hole at one end (the pipe is intended to be a closed reservoir) and methane with assumption of ideal gas was considered as natural gas. Modeling of [13] was assumed as one-dimensional and the flow in the pipe as adiabatic. In addition, the finite difference method (the Secant iterative algorithm) was used to solve the governing equations of the problem.

The model which has used for validation in this paper contains a pipe that a hole has been created at the end of it. Boundary conditions and solution assumptions for this part of simulation are considered similar to the Montiel's work [13].

Fig. 5 shows the comparison between results of [13] and the model of this study. As expected, in this case the amount of gas leakage is larger compared [13] model and it is due to reversal flow from downstream toward upstream. According to Fig. 5, the value of minimum and maximum percentage of relative difference between results of two models to result of Montiel's model [13] is $7 \%$ and $28 \%$, respectively. 


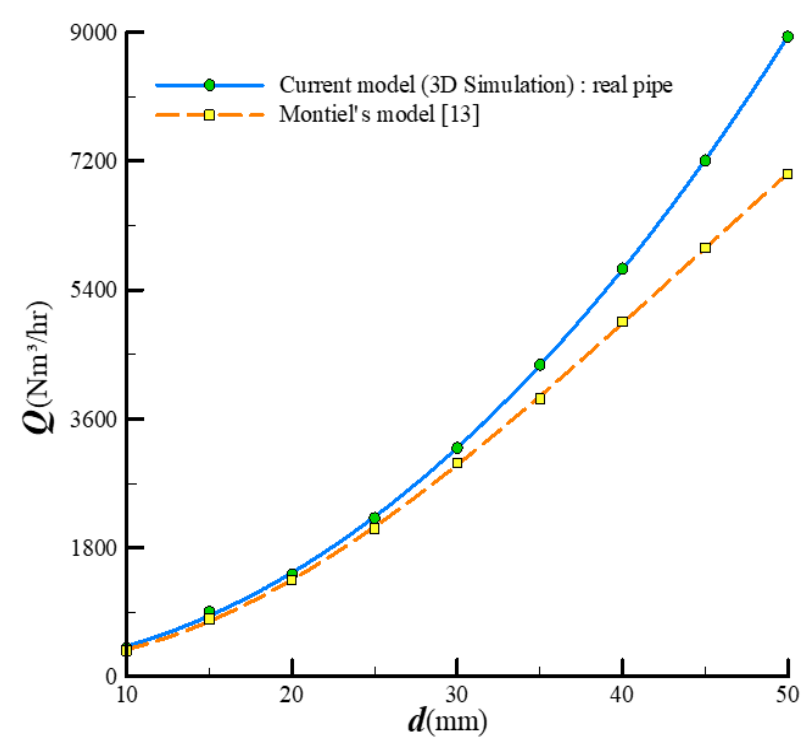

Fig. 5: Comparison of gas leakage between current model and Montiel's model [13]

\section{$B$. Investigating the effect of the parameters}

Fig. 6 illustrates the volumetric flow rate changes in terms of the hole diameter (d) and inlet pressure $\left(\mathrm{p}_{1}\right)$. Based on this figure it can be concluded that the leakage volumetric flow rate has a linear and second-order direct relationship with pipe diameter and hole diameter, respectively. Also by increasing the initial point pressure, the pressure difference of initial point and the hole gets more and as a result the leaked gas flow from the hole increases.

Analyzing the results shows that considering the length of pipe after the hole show its impact on large diameter holes (due to increasing the amount of the reversal flow by increasing the hole size). In other words, disregarding length of downstream in past studies causes the results go away from actual condition.

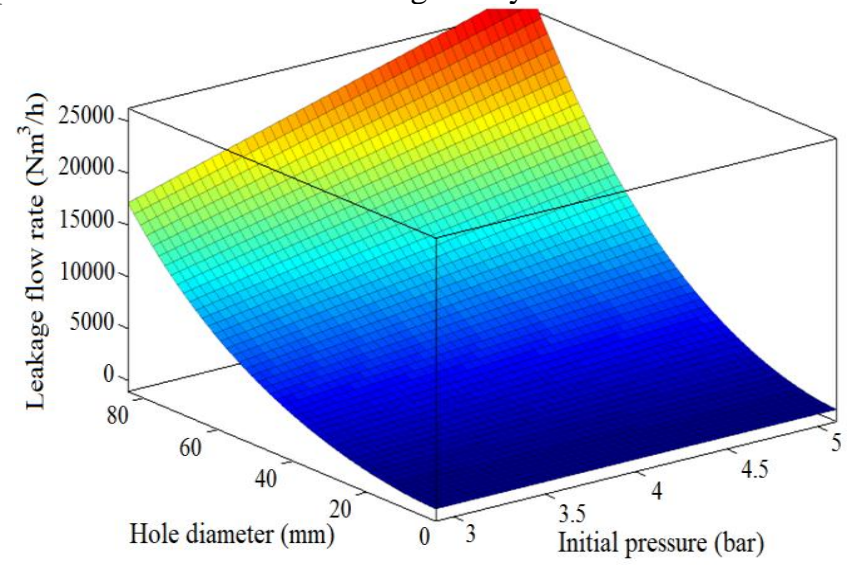

Fig. 6: Volumetric flow rate of leakage as a function of hole diameter and pressure of initial point for $\mathrm{D}=114.6 \mathrm{~mm}$

Fig. 7 displays the absolute pressure contour plot. As it can be seen in this figure, the pressure changes along the pipe is very low and there is a significant pressure variation only around the hole; this is due to the low pressure level of urban distribution NG pipelines.

A sample of the velocity magnitude distribution inside the pipe and around the hole is drawn in Fig. 8. Since the small holes have been investigated in current work, the average of flow velocity reaches to the sound speed at the hole and according to the Fig. 9, the average of Mach number at the hole is unit.

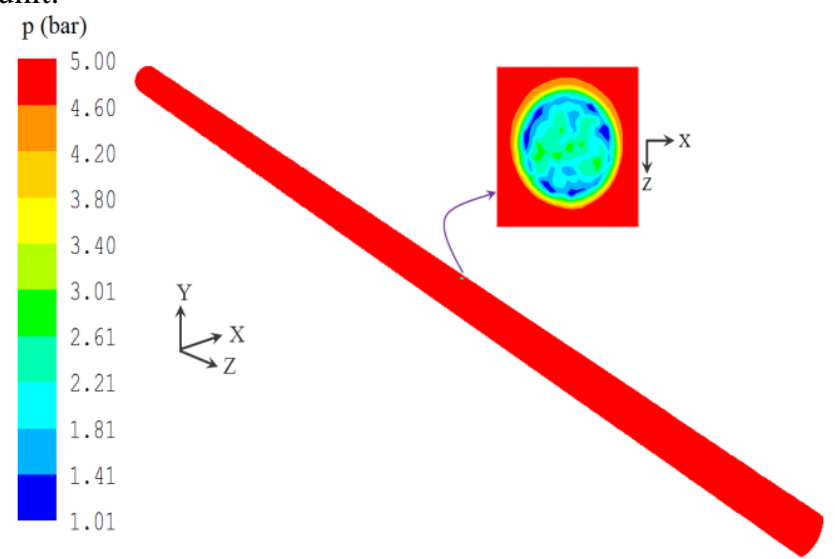

Fig. 7: Absolute pressure contour for $\mathrm{d}=20 \mathrm{~mm}, \mathrm{D}=114.6 \mathrm{~mm}$ and

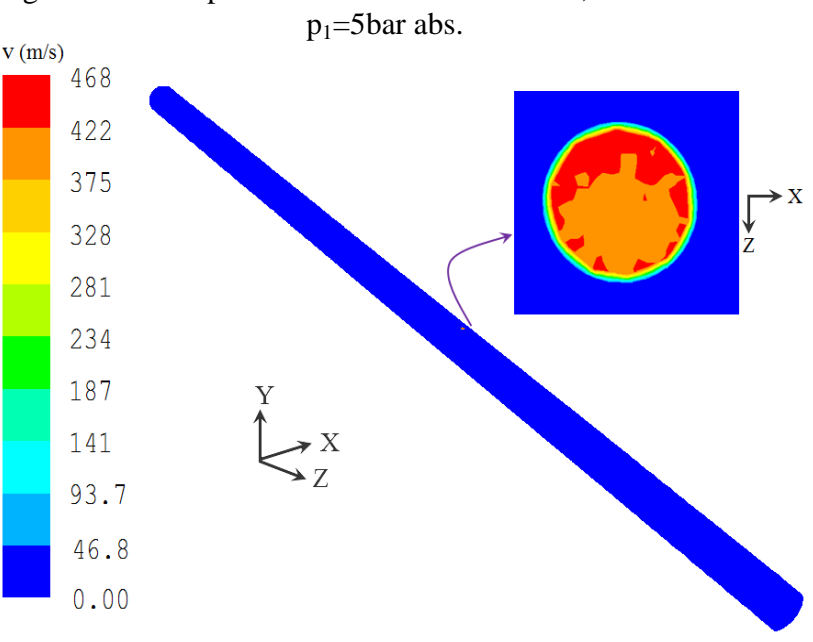

Fig. 8: Velocity contour for $\mathrm{d}=20 \mathrm{~mm}, \mathrm{D}=114.6 \mathrm{~mm}$ and $\mathrm{p}_{1}=5$ bar

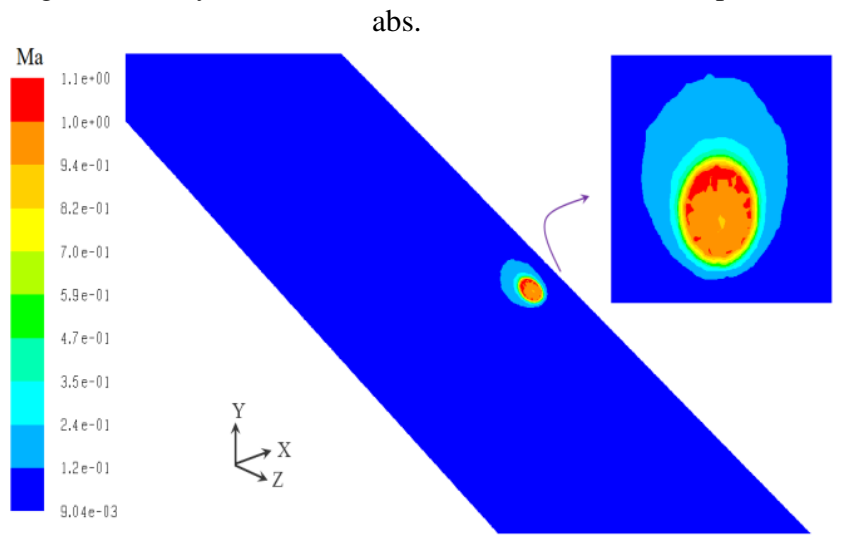

Fig. 9: Mach number contour for $\mathrm{d}=20 \mathrm{~mm}, \mathrm{D}=114.6 \mathrm{~mm}$ and $\mathrm{p}_{1}=5$ bar abs. 


\section{CONCLUSION}

In this paper has been paid to simulate NG leakage scenario by using three-dimensional numerical modeling with Ansys-Fluent software. The flow is considered turbulent and compressible and the pipeline is located in low pressure distribution pipelines. Accordingly, the pressure change along the pipe is very low and just there are pressure changes around the hole. The flow velocity condition at the hole is a sonic flow and the outlet velocity reaches to the sound speed. By analyzing the results, it can be stated that by increasing hole diameter, the pressure difference between the ambient pressure and the damaged section increases and the pressure difference between the initial point pressure and the damaged section decreases. According to the results, the volumetric flow rate of NG leakage changes as ascending functions approximately into the second and first orders with hole diameter and initial point pressure, respectively.

\section{ACKNOWLEDGMENT}

This study was funded by Young Researchers and Elite Club, Mashhad Branch, Islamic Azad University, Mashhad, Iran.

\section{REFERENCES}

[1] Arpino, F., Dell'Isola, M., Ficco, G., Vigo, P., 2014. "Unaccounted for gas in natural gas transmission networks: Prediction model and analysis of the solutions". Journal of Natural Gas Science and Engineering 17, $58-70$. https://doi.org/10.1016/j.jngse.2014.01.003

[2] Costello, K.W., 2014. "Lost and unaccounted-for gas: Challenges for public utility regulators". Utilities Policy 29, 17-24. https://doi.org/10.1016/j.jup.2014.04.003

[3] Metro, Paul., 2007. "Technical losses in natural gas transportation, distribution, and storage". Presentation to the Energy Agency of the Republic of Serbia, Available at: http://www.naruc.org/international/Documents/Technical_losses_in_nat ural_gas_transportation_distribution_storage_Paul_Metro.pdf.

[4] Zhang, T., Tan, Y., Zhang, X., Zhao, J., 2015. "A novel hybrid technique for leak detection and location in straight pipelines". Journal of Loss Prevention in the Process Industries 35, 157-168. https://doi.org/10.1016/j.jlp.2015.04.012

[5] Parvini, M., Gharagouzlou, E., 2015. "Gas leakage consequence modeling for buried gas pipelines". Journal of Loss Prevention in the Process Industries 37, 110-118. https://doi.org/10.1016/j.jlp.2015.04.012

[6] Ebrahimi-Moghadam, A., Farzaneh-Gord, M., Deymi-Dashtebayaz, M., 2016. "Correlations for estimating natural gas leakage from above-ground and buried urban distribution pipelines". Journal of Natural Gas Science and Engineering 34, 185-196.

[7] Nouri-Borujerdi, A., Ziaei-Rad, M., 2009. "Simulation of compressible flow in high pressure buried gas pipelines". International Journal of Heat and Mass Transfer 52, 5751-5758.

https://doi.org/10.1016/j.ijheatmasstransfer.2009.07.026

[8] Liu, C., Li, Y., Meng, L., Wang, W., Zhao, F., Fu, J., 2015. "Computational fluid dynamic simulation of pressure perturbations generation for gas pipelines leakage". Computers \& Fluids 119, 213-223. https://doi.org/10.1016/j.compfluid.2015.06.023

[9] Moloudi, R., Abolfazli Esfahani, J., 2014. "Modeling of gas release following pipeline rupture: Proposing non-dimensional correlation". Journal of Loss Prevention in the Process Industries 32, 207-217. https://doi.org/10.1016/j.jlp.2014.09.003

[10] Lu, L., Zhang, X., Yan, Y., Li, J.M., Zhao, X., 2014. “Theoretical analysis of natural-gas leakage in urban medium-pressure pipelines". Journal of Environment and Human 1, 71-86. https://doi.org/10.15764/EH.2014.02009

[11] Kostowski, W.J., Skorek, J., 2012. "Real gas flow simulation in damaged distribution pipelines". Energy 45, 481-488. https://doi.org/10.1016/j.energy.2012.02.076

[12] Keith, J.M., Crowl, D.A., 2005. "Estimating sonic gas flow rates in pipelines". Journal of Loss Prevention in the Process Industries 18(2), 55-62. https://doi.org/10.1016/j.jlp.2004.12.004

[13] Montiel, H., Vilchez, J., Casal, J., Arnaldos, J., 1998. "Mathematical modeling of accidental gas releases". Journal of Hazardous Materials 59, 211-233. https://doi.org/10.1016/S0304-3894(97)00149-0

[14] Cavazzuti, M., Corticelli, M.A., 2017. "Numerical modelling of Fanno flows in micro channels: a quasi-static application to air vents for plastic moulding". Thermal Science and Engineering Progress 2, 43-56. https://doi.org/10.1016/j.tsep.2017.04.004

[15] Oke, A., Mahgerefteh, H., Economou, I., Rykov, Y., 2003. "A transient outflow model for pipeline puncture". Chemical Engineering Science 58, 4591-4604. https://doi.org/10.1016/S0009-2509(03)00338-5

[16] Jo, Y., Ahn, B., 2003. "A simple model for the release rate of hazardous gas from a hole on high-pressure pipelines". Journal of Hazardous Materials 97, 31-46.

[17] Luo, J.L., Zheng, M., Zhao, X.W., Huo, C.Y., Yang, L., 2006. "Simplified expression for estimating release rate of hazardous gas from a hole on high-pressure pipelines". Journal of Loss Prevention in the Process Industries 19, 362-366.

[18] Liu, C., Li, Y., Meng, L., Wang, W., Zhao, F., Fu, J., 2015. "Computational fluid dynamic simulation of pressure perturbations generation for gas pipelines leakage". Computers \& Fluids 119, 213-223. https://doi.org/10.1016/j.compfluid.2015.06.023

[19] Detemmerman, T., Froment, F., 1998. "Three Dimensional Coupled Simulation of Furnaces and Reactor Tubes for the Thermal Cracking of Hydrocarbons". Oil and Gas Science and Technology - Rev. IFP, 53(2), 181-194. https://doi.org/10.2516/ogst:1998017

[20] NoorAliPour Nahavandi, N., Farzaneh-Gord, M., 2013. "Numerical simulation of filling process of natural gas onboard vehicle cylinder". Journal of the Brazilian Society of Mechanical Sciences and Engineering, 35, 247-256, DOI: 10.1007/s40430-013-0020-3. https://doi.org/10.1007/s40430-013-0020-3

[21] Nagaosa, R.S., 2014. "A new numerical formulation of gas leakage and spread into a residential space in terms of hazard analysis". Journal of Hazardous Materials 271, 266-274. https://doi.org/10.1016/j.jhazmat.2014.02.033

[22] Handbook of Polyethylene Pipe, second ed. Plastics Pipe Institute (PPI), Decker Court. 